\title{
Radial Correlation Length Measurements on ASDEX Upgrade Using Correlation Doppler Reflectometry
}

\author{
J Schirmer, G D Conway, E Holzhauer*, W Suttrop, H Zohm and the \\ ASDEX Upgrade Team \\ Max-Planck-Institut für Plasmaphysik, EURATOM-Association IPP, D-85748 Garching, \\ Germany \\ *Institut für Plasmaforschung, Universität Stuttgart, D-70569 Stuttgart, Germany
}

\begin{abstract}
The technique of correlation Doppler reflectometry for providing radial correlation length $L_{r}$ measurements is explored in this paper. Experimental $L_{r}$ measurements are obtained using the recently installed dual channel Doppler reflectometer system on ASDEX Upgrade. The experimental measurements agree well with theory and with $L_{r}$ measured on other fusion devices using different diagnostic techniques. A strong link between $L_{r}$ and plasma confinement could be observed. During an L-H transition, an increase in the absolute value of $E_{r}$ shear was detected at the same plasma edge region where a decrease in $L_{r}$ was measured. This observation is in agreement with theoretical models which predict that an increase in the absolute shear suppresses turbulent fluctuations in the plasma, leading to a reduction in $L_{r}$. Furthermore, $L_{r}$ decreases from plasma core to edge due to the more highly confined plasma pedestal region. Measurements of $L_{r}$ versus plasma triangularity $\delta$ were also obtained, showing a decrease of $L_{r}$ with increasing $\delta$. This indicates that plasma confinement is improving with triangularity. In connection with the experimental results, an investigation of the correlation Doppler reflectometer response function using a 2-dimensional finite difference time domain (FDTD) code was performed. The simulation results confirm that Doppler reflectometry provides robust radial correlation lengths of the turbulence with high resolution and suggests that $L_{r}$ is independent of the turbulence wavenumber $k_{\perp}$ and its fluctuation level.
\end{abstract}

Submitted to: Plasma Phys. Control. Fusion

PACS numbers: 52.55.Fa, 52.35.Ra, 52.70.Gw 


\section{Introduction}

One of the major goals of the tokamak fusion program is the understanding and control of plasma turbulence. Turbulence causes additional radial transport of heat and particles, thereby degrading the overall confinement of the plasma. A new diagnostic technique, Doppler reflectometry, has been developed for measurements of plasma rotation profiles and turbulence properties. It is a type of microwave radar technique which uses the back-scatter of microwaves from a radial position in the plasma where the refractive index equals zero. In this paper, the technique is extended for turbulence correlation measurements by adding a second Doppler reflectometer channel such that two microwave beams are launched from the same antenna into the plasma with a small frequency difference. A radial correlation Doppler reflectometer system can provide simultaneous measurements of the plasma radial electric field $E_{r}$ and its shear $\delta E_{r} / \delta r$ (both parameters are believed to be fundamental for suppressing turbulence) together with measurements of the properties of the plasma turbulence, such as the radial correlation length of the turbulence $L_{r}$.

The radial correlation length of the turbulence, $L_{r}$, has in the past been measured with a variety of diagnostics, the most common being standard correlation reflectometry $[1,2,3,4,5]$. However, there has been divergence concerning not only how $L_{r}$ is determined but also how it is defined $[6,7,8,9]$. The measurement of $L_{r}$ is valuable since it provides the spatial size of a turbulent perturbation (i.e. eddy). In this paper, the technique of correlation Doppler reflectometry for $L_{r}$ measurements is explored. The paper begins with a description of the diagnostic technique followed by a series of example $L_{r}$ measurements made on the ASDEX Upgrade tokamak. The measurements are compared with theory and with correlation lengths measured in other tokamaks using standard correlation reflectometry. In addition, a 2D Finite Difference Time Domain code was adapted to simulate $L_{r}$ measurements under realistic ASDEX Upgrade plasma conditions in order to establish how well the diagnostic determines the true turbulent $L_{r}$. The simulation results are presented in section 4 .

\section{Correlation Doppler Reflectometry: Measurement Technique}

Doppler reflectometry is a diagnostic technique based on the back-scatter of microwaves close to the plasma cut-off position. Having first been introduced as a plasma diagnostic around 1998 for measurements of the plasma rotation velocity $[10,11]$ and the radial electric field $\left(E_{r}\right)$ [12], the technique has been further developed for measurements of the radial electric field shear $\left(\delta E_{r} / \delta r\right)$ [12] and the radial correlation length of the turbulence $\left(L_{r}\right)$ [13]. The principle behind the technique is explained in references $[10,11]$. The main difference to standard fluctuation reflectometry is that the microwave beam in the Doppler reflectometry technique is launched from a transmitting antenna into the plasma at a finite tilt angle $\theta_{\text {tilt }}$ with respect to the normal to the cutoff surface. The incident beam is both reflected and Bragg scattered at a corrugated cutoff layer in the plasma. The position of the cutoff (i.e. point of reflection in the plasma) is determined as the position where the index of refraction equals zero. It differs depending on whether $\mathrm{O}$-mode or $\mathrm{X}$-mode polarization of the microwave is 
selected $[14,12]$. The backscattered reflected signal from the plasma cutoff is then detected by a nearby antenna. Assuming a monostatic system (valid since the receiving and transmitting antenna are displaced toroidally while their inclination is poloidal), the geometry selects the diffraction pattern of mainly order $m=-1$. As a result, the Bragg diffraction equation gives the condition for slab geometry

$$
k_{\perp}=2 k_{\circ} \sin \theta_{\text {tilt }}
$$

where $k_{\circ}=2 \pi / \lambda_{\circ}$ is the probing wavenumber. Equation 1 shows that by varying the tilt angle, the Doppler reflectometer selects plasma density perturbations with finite wavenumber $k_{\perp}$ in the cutoff layer by the Bragg condition. Therefore, the diagnostic can provide access to the $k$ spectrum of the turbulence.

In a fluctuating plasma, moving with velocity $u_{\perp}$, a Doppler shift is detected in the received microwave signal. Along field aligned fluctuations (i.e. $k_{\|}=0$ ), the Doppler shift results only from the velocity component of the perturbations perpendicular to the magnetic field [10] and is given by:

$$
f_{D}=\frac{\omega_{D}}{2 \pi}=\frac{k_{\perp} u_{\perp}}{2 \pi}
$$

where $f_{D}$ is the Doppler frequency shift. On ASDEX Upgrade, typical values of $\theta_{\text {tilt }}$ range between 15 to $25^{\circ}$ depending on the plasma shape. The measured Doppler shifts $f_{D}$ can range anywhere between +2000 to $-4000 \mathrm{kHz}$, giving $u_{\perp}$ values between +20 to $-40 \mathrm{~km} / \mathrm{s}$.

The velocity $u_{\perp}$ contains two components:

$$
u_{\perp}=v_{E \times B}+v_{p h}
$$

where $v_{E \times B}$ is the $E \times B$ velocity and $v_{p h}$ is the phase velocity of the density fluctuation or turbulence moving in the plasma frame $\left(E_{r}=0\right)$. Generally, for edge conditions $v_{p h}$ is negligible. Both numerical calculations and experiment indicate that $v_{E \times B}$ dominates for drift wave turbulence at the plasma edge (see [12] and references therein as well as [15]). As a result, equation 3 reduces to $u_{\perp} \approx v_{E \times B}$, from which $E_{r}$ can be calculated:

$$
E_{r}=-u_{\perp} B
$$

Typically, a complete radial profile of $E_{r}$ is measured in $100 \mathrm{~ms}$ using a staircase stepped launch frequency pattern from 50 to $74 \mathrm{GHz}$. The errors in the $E_{r}$ measurement arise from the calculation of the tilt angle and the determination in $f_{D}$. The error in the radial position depends on the quality of the density profile.

With the introduction of a second Doppler reflectometer, a correlation Doppler reflectometry system may be constructed. On ASDEX Upgrade, two heterodyne V-band Doppler reflectometers are connected to the same antenna pair so that they launch microwaves with the same line of sight simultaneously into the plasma. The microwaves have different launch frequencies $\left(f_{1}\right.$ and $\left.f_{2}\right)$ and therefore reflect from different radial positions in the plasma $\left(r_{1}\right.$ and $\left.r_{2}\right)$. Further details describing the hardware may be found in references $[12,16]$.

To obtain $\partial E_{r} / \partial r$ measurements, the launch frequencies of the microwave beams are repetitively swept with a constant fixed frequency difference $\triangle f=f_{2}-f_{1}$ such that two 


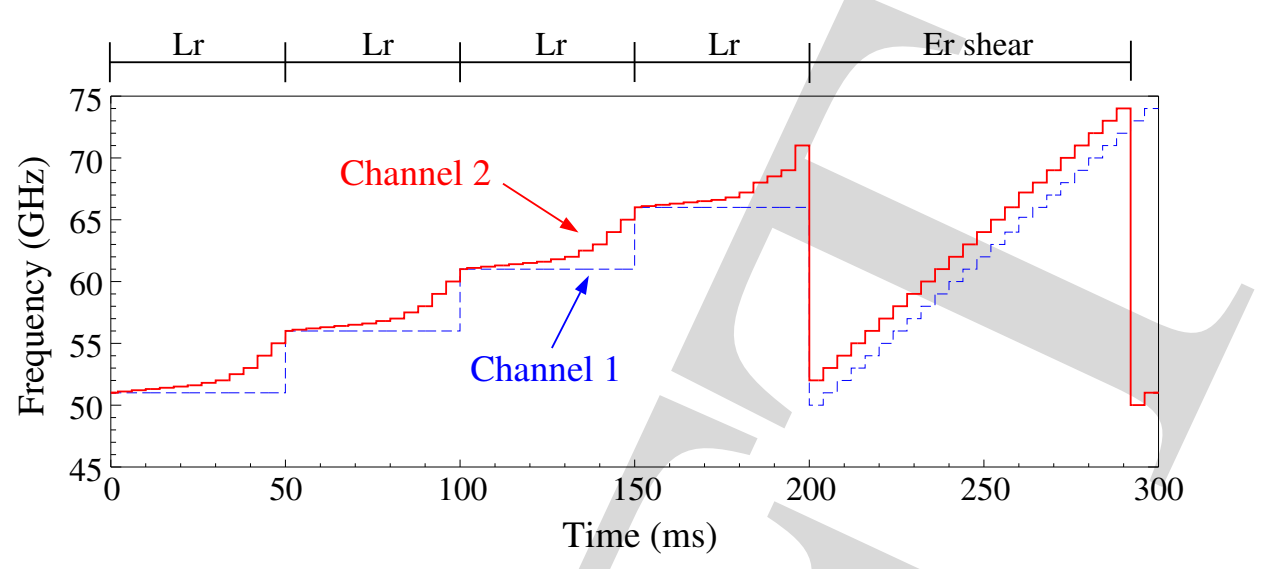

Figure 1. Typical frequency patterns used for simultaneous $E_{r}$ shear and $L_{r}$ measurements.

simultaneous radial profiles of $E_{r}$ can be constructed from the Doppler frequency shift in the reflectometer signal. Taking the difference between the two channels' $E_{r}$ values measured at the same time divided by their radial separation gives a radial profile of the instantaneous $E_{r}$ shear $\left(\partial E_{r} / \partial r\right)$. Typically, a frequency sweep pattern of $1 \mathrm{GHz}$ steps from 50 to $74 \mathrm{GHz}$ in $100 \mathrm{~ms}$ with a fixed $2 \mathrm{GHz}$ separation is used for these measurements as shown in figure 1 between 200 and 300ms. At the plasma edge, a $2 \mathrm{GHz}$ separation corresponds to approximately a $1 \mathrm{~cm}$ radial separation.

Alternatively, keeping the frequency of one reflectometer channel $f_{1}$ constant and sweeping the frequency of the second $f_{2}$ permits the correlation properties of the turbulence to be measured. Here, a stair case every $50 \mathrm{~ms}$ with a frequency difference between the two channels starting at $0.1 \mathrm{GHz}$ (to avoid any cross talk between the two channels) and increasing logarithmically was found to be appropriate. Figure 1 illustrates in the first $200 \mathrm{~ms}$ four of these frequency patterns covering the V-band range. Cross correlating the two Doppler shifted reflectometer signals gives the coherence between the two signals as a function of their frequency separation. The density profile $n_{e}$ is required to translate the frequency separation $\triangle f$ to a radial separation $\Delta r$. The radial separation when the coherence drops to 1/e gives a measure of the spatial correlation of the turbulence (designated the radial correlation length, $L_{r}$ ) for the particular $k_{\perp}$ probed.

The cross correlation is a measure of similarity of two signals. It is given by the cross correlation function [17]:

$$
R_{x y}(\tau)=\int_{0}^{t} x(t) y(t+\tau) d t
$$

where $\tau$ is the time lag between the two signals. The auto correlation functions are given by

$$
\begin{aligned}
& R_{x x}(\tau)=\int_{0}^{t} x(t) x(t+\tau) d t \\
& R_{y y}(\tau)=\int_{0}^{t} y(t) y(t+\tau) d t
\end{aligned}
$$

From Equations 5, 6 and 7, the correlation coefficient $\rho_{x y}(\tau)$ may be obtained. It is defined as

$$
\rho_{x y}(\tau)=\frac{R_{x y}(\tau)}{\left(R_{x x}(0) R_{y y}(0)\right)^{1 / 2}}
$$



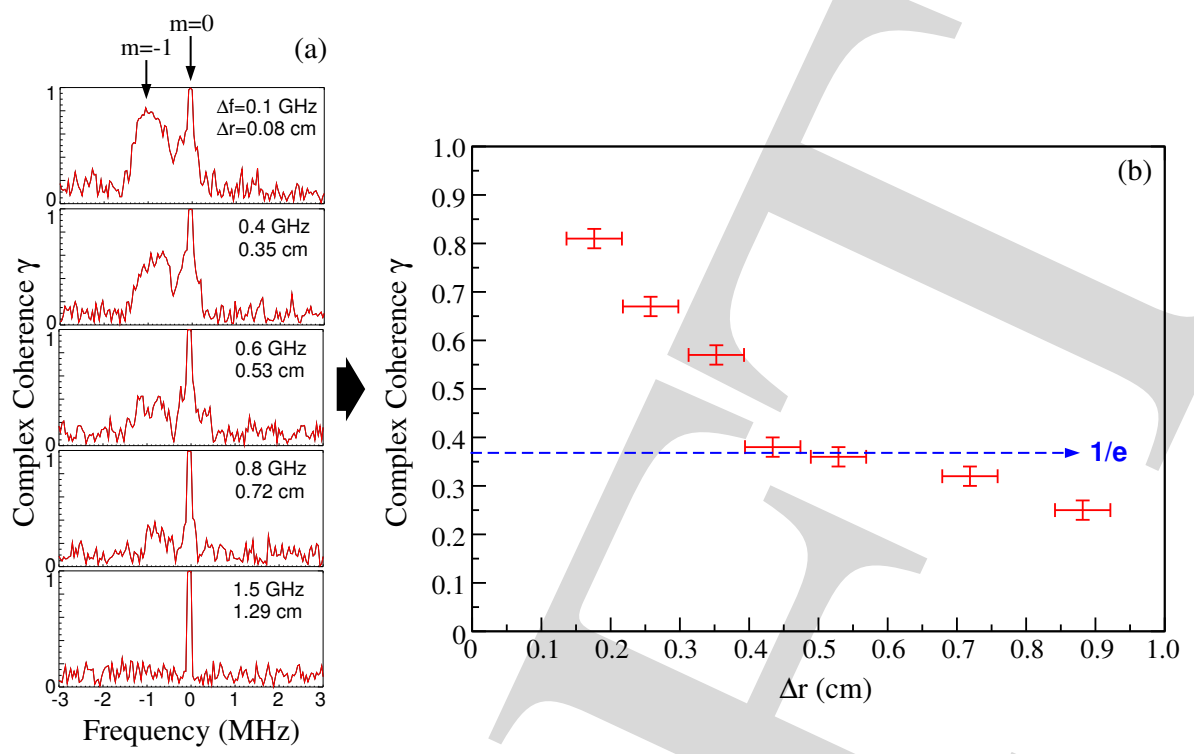

Figure 2. (a) Coherence spectra for various $\triangle f$ and (b) coherence versus $\triangle r$ for L-mode USN discharge \# 19148 at time $=2.95$ s to 3.00s. Data averaged over $2 \mathrm{~ms}$. FFT window 512 points.

The correlation coefficient measures the degree of linear dependence between $x(t)$ and $y(t)$ for time lag $\tau$. By taking the fast Fourier transform (FFT) of $\rho_{x y}(\tau)$, the coherence as a function of frequency $\gamma(f)$ is obtained. The coherence $\gamma(f)$ can be calculated by

$$
\gamma(f)=\frac{\left\langle S_{x y}(f)\right\rangle}{\left(\left\langle S_{x x}(f)\right\rangle\left\langle S_{y y}(f)\right\rangle\right)^{1 / 2}}
$$

where $S_{x y}, S_{x x}$ and $S_{y y}$ are the cross and auto spectra computed from the Fourier transform of the complex signals, $X(f)$ and $Y(f)$. The brackets \langle\rangle represent ensemble averages over many data sets. The coherence ranges from zero to one and is equal to unity when the signals are identical. Ideally, the coherence should drop to zero as the signals become more different. However due to the limited signal lengths, the coherence has a statistical minimum of $\gamma=1 / \sqrt{N}$ where $N$ is the number of ensembles. Figure 2 shows on the left hand side coherence spectra for increasing $\triangle f$ obtained by cross correlating the complex Doppler reflectometer signals $\left(A e^{i \phi}\right)$. As can be seen from the coherence spectra in figure 2(a), a peak in the coherence is obtained at the same frequency as the Doppler shift. This peak decreases with increasing frequency difference as expected. The peak values are plotted in figure 2(b) as a function of radial separation and the 1/e coherence value is designated as the radial correlation length, $L_{r}$.

There is some debate over the best type of reflectometer signal used to determine radial correlation lengths as well as in how the $L_{r}$ lengths are defined [7]. Figure 3 shows coherence versus $\Delta r$ for the homodyne $(I=A \cos \phi$ and $Q=A \sin \phi)$, amplitude $(A)$, phase $(\phi)$ and complex $\left(I+i Q=A e^{i \phi}\right)$ signals measured during an L-mode discharge at $\rho_{\text {pol }} \approx 0.92$. For all $L_{r}$ measurements on ASDEX Upgrade, the complex and homodyne signals consistently give larger $L_{r}$ than the amplitude and phase signals as seen in figure 3. Estrada also investigated 


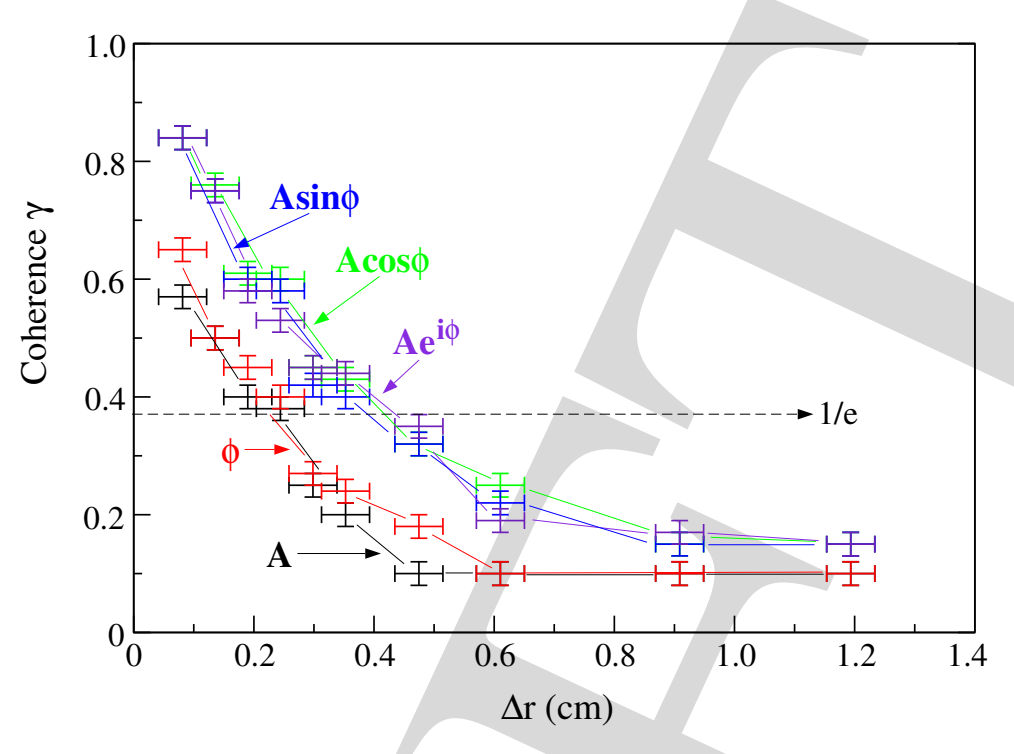

Figure 3. Radial coherence curves for a variety of Doppler fluctuation signals measured in L-mode discharge \#19148 (2.35-2.40s) using X-mode polarization.

this subject and found that the homodyne signals performed more reliably than the amplitude and phase signals [8]. Rhodes has also shown by experiments that plasma turbulence is most adequately represented by homodyne signals [18] as has Gilmore [4] and by modelling Conway [7]. This is most likely due to the fact that the homodyne and complex signals include both amplitude and phase information and therefore do not assign too much weight to either parameter, particularly during periods of low reflected power. All $L_{r}$ measurements presented in this paper are obtained from analysis using complex Doppler reflectometer signals.

The error in $L_{r}$ measurements has several sources. First, in the presence of strong, rapidly changing $E_{r}$ shear, the frequency scales are contracted/expanded in the neighbouring layers. In such cases, caution in the interpretation of the data is required and typically measurements of $L_{r}$ under these circumstances is avoided. Second, the coherence error depends on the data length (i.e. number of fast Fourier transform FFT points and number of ensemble averages). Since the number of data points is fixed by the data acquisition, there is a compromise between spectral resolution and statistical error in the coherence. The uncertainty in the coherence is usually taken as \pm 0.02 (i.e. $1 / \sqrt{N}$ ). Third, the determination of the cutoff layer radius requires a reliable density profile. The typical error in $r$ is $0.02 \mathrm{~cm}$ resulting in an error of $0.04 \mathrm{~cm}$ in $\Delta r$.

\section{Radial Correlation Length Measurements}

In this section, several examples of experimental $L_{r}$ measurements are presented and then compared with theory and contrasted with $L_{r}$ measurements made on other machines using different diagnostics. 


\subsection{Dependence on Plasma Heating}

During Ohmic (1.5 - 1.6s), L-mode ECRH heated (2.5 - 2.6s) and L-mode NBI heated phases $(6.3-6.4 \mathrm{~s})$ of discharge $\# 18707\left(B_{\phi}=-2.3 \mathrm{~T}, I_{p}=+0.8 \mathrm{MA}\right)$, profiles of $E_{r}$ and $L_{r}$ were measured simultaneously and are shown in figure 4 . The plasma electron density during these phases was constant at $\overline{n_{e}} \approx 3.6 \times 10^{19} \mathrm{~m}^{-2}$. The $E_{r}$ profiles, shown in figure $4($ a), are plotted
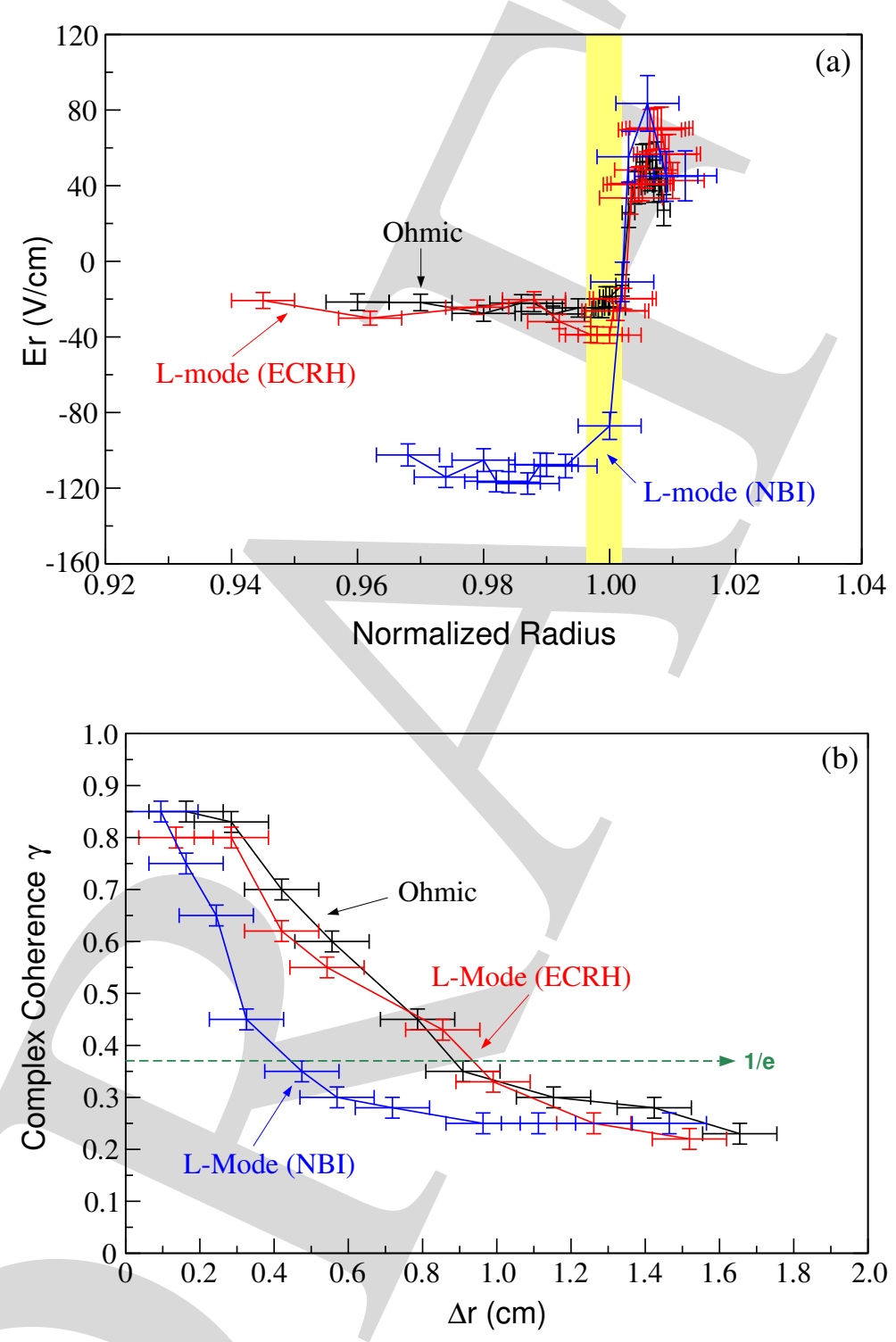

Figure 4. (a) $E_{r}$ profiles and (b) corresponding $L_{r}$ measurements obtained during discharge \#18707 using $X$-mode polarization. The shaded region displays the radial position where $L_{r}$ was measured. During the NBI heated phase, the edge $E_{r}$ becomes more negative and the radial correlation length decreases.

versus normalized poloidal flux radius $\left(\rho_{p o l}\right)$, which is defined as

$$
\rho_{p o l}=\sqrt{\frac{\Psi-\Psi_{a}}{\Psi_{s}-\Psi_{a}}}
$$


where $\Psi$ is the poloidal flux and the subscripts $s$ and $a$ denote the separatrix and the magnetic axis respectively. In the Scrape Off Layer (SOL), where $\rho_{\text {pol }}>1$, radial electric fields reaching $+60 \pm 20 \mathrm{Vcm}^{-1}$ are measured. $E_{r}$ is positive due to the fast thermal motion of parallel electron losses [19]. At the plasma edge $\left(0.90<\rho_{\text {pol }}<1.00\right)$, the $E_{r}$ profiles undergo a reversal and a negative $E_{r}$ well is formed. The minimum $E_{r}$ measured in L-modes and Ohmic discharges ranges between -50 and $-100 \mathrm{Vcm}^{-1}$ while in $\mathrm{H}$-mode discharges, these fields decrease dramatically ranging anywhere between -300 to $-450 \mathrm{Vcm}^{-1}$ [12]. Note the edge $E_{r}$ profile becomes more negative during the NBI heated phase in figure 4 due to the increasing pressure gradient at the edge pedestal. The increasing gradient raises the diamagnetic velocity, which is one of the main contributors of $E_{r}$ at the plasma edge. Figure 4 shows that in the shaded region, an increase in $\left|E_{r}\right|$ is linked to a decrease in $L_{r}$. Hence, NBI heating is observed to decrease the edge radial correlation lengths in comparison with ECRH heating. Furthermore, the H98 factor is higher in thr NBI phase, indicating the improved plasma confinement.

\subsection{The L-H transition}

There has been effort from both the experimental and theoretical side to examine the relationship between the radial electric field, its shear and $L_{r}$. The Biglari Diamond and Terry (BDT) model [20], for example, claims that an increase in absolute $E_{r}$ shear suppresses density fluctuations, stabilizes the turbulence and decreases $L_{r}$. This could be verified in the following measurement. Figure 5 shows $E_{r}$ shear profiles and $L_{r}$ measurements during Lmode and H-mode phases of discharge \#19151. The $E_{r}$ shear profiles are positive around the SOL region, becoming negative in the pedestal region. Previous $E_{r}$ shear measurements on ASDEX Upgrade indicate that the positive shear associated with the SOL region is relatively constant for a variety of discharges while the negative shear associated with the plasma pedestal region changes [12]. The edge $E_{r}$ shear in the H-mode phase in this example reaches a minimum of $-(290 \pm 30) \mathrm{Vcm}^{-2}$ while in L-mode, it reaches only $-(42 \pm 5) \mathrm{Vcm}^{-2}$. These values are typical of L-mode and H-mode discharges on ASDEX Upgrade $[12,16]$. Around this region of negative shear, at $\rho_{\text {pol }} \approx 0.90$, an $L_{r}$ of $(0.37 \pm 0.04) \mathrm{cm}$ in H-mode and $(0.88 \pm 0.04) \mathrm{cm}$ in L-mode is measured. As expected, the radial correlation length reduces with improved confinement and increasing $\left|\partial E_{r} / \partial r\right| . L_{r}$ decreases by a factor of two in $\mathrm{H}-$ modes. A similar reduction was reported on the DIII-D tokamak using standard correlation reflectometry [21].

\section{3. $L_{r}$ as a function of normalized radius, $\rho_{p o l}$}

$E_{r}$ shear measurements have shown, as in figure 5, large varying shear values at the plasma edge but dropping to negligible values in the mid-core region [12]. From the Biglari, Diamond and Terry model, one would expect that the radial correlation lengths also reflect a similar trend, such that in the regions of high shear at the plasma edge, $L_{r}$ is small and in regions of low shear in the plasma core, $L_{r}$ is large. 

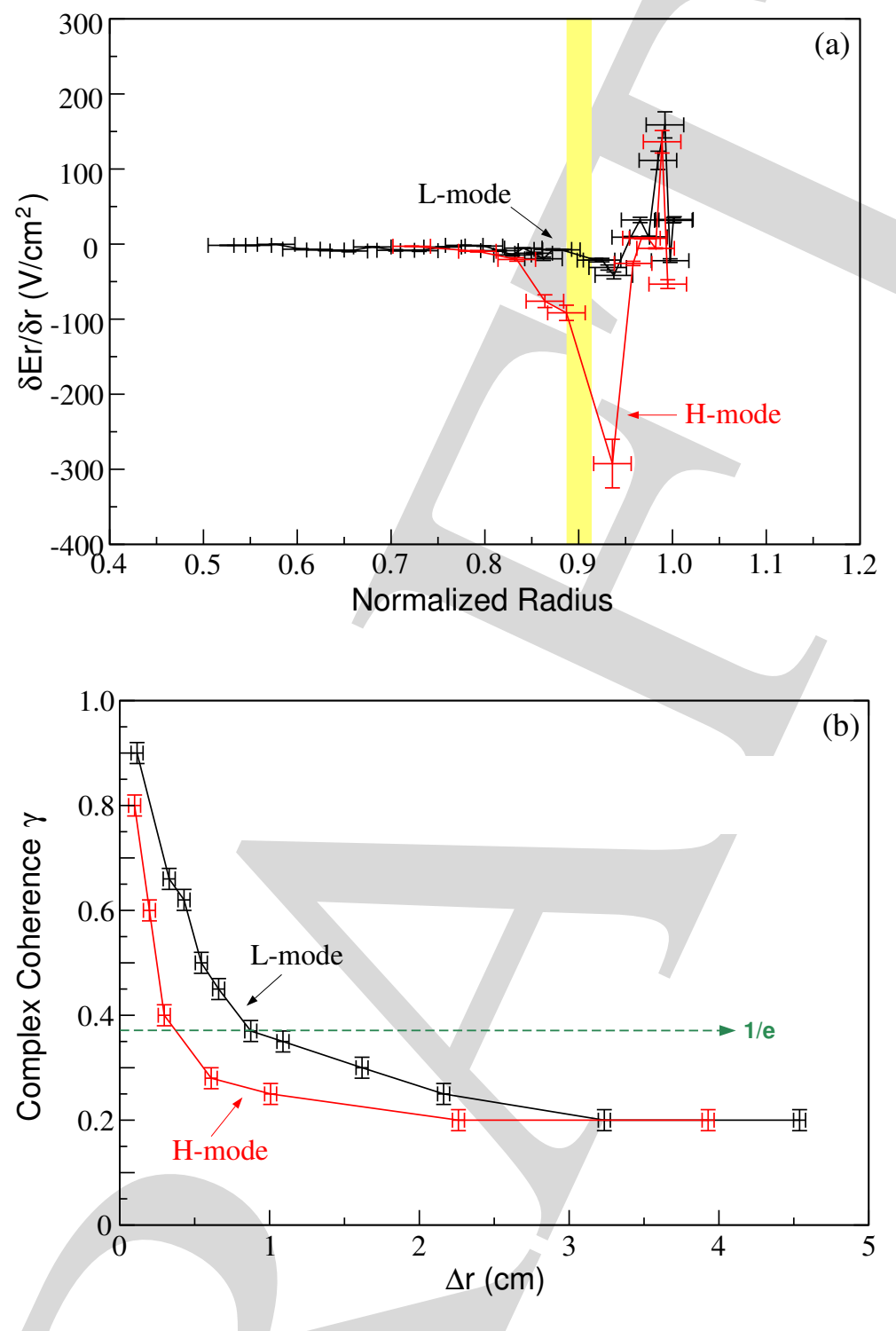

Figure 5. The simultaneous measurement of (a) $E_{r}$ shear and (b) $L_{r}$ in an L-H transition discharge (\#19151) using O-mode polarization. $L_{r}$ was measured at $\rho_{\text {pol }} \approx 0.90 \pm 0.02$, marked by the yellow shaded region. The result shows a reduction in $L_{r}$ in the region of high $E_{r}$ shear.

Figure 6 shows how $L_{r}$ varies with radial position. The data are for constant L-mode conditions with $B_{\phi}=-2.0 \mathrm{~T}, I_{p}=+1.0 \mathrm{MA}$ and $2.5 \mathrm{MW}$ of NBI heating power. Note that the radial positions of the measurements were corrected using the TORBEAM beam-tracing code [22] to account for the beam refractive effects in the plasma, particularly in the core. The figure shows $L_{r}$ increasing into the plasma core, ranging from about $0.71 \mathrm{~cm}$ at $\rho_{\text {pol }} \approx$ 0.95 to $2.89 \mathrm{~cm}$ at $\rho_{\text {pol }} \approx 0.58$. The magnitude of $L_{r}$ is similar to those measured on DIII-D using a standard correlation reflectometer $\left(k_{\perp}=0\right)$ [2]. The increase of $L_{r}$ into the plasma core suggests its link to plasma confinement and turbulence. $L_{r}$ is smaller in the better confined pedestal region of the plasma as opposed to the core. 


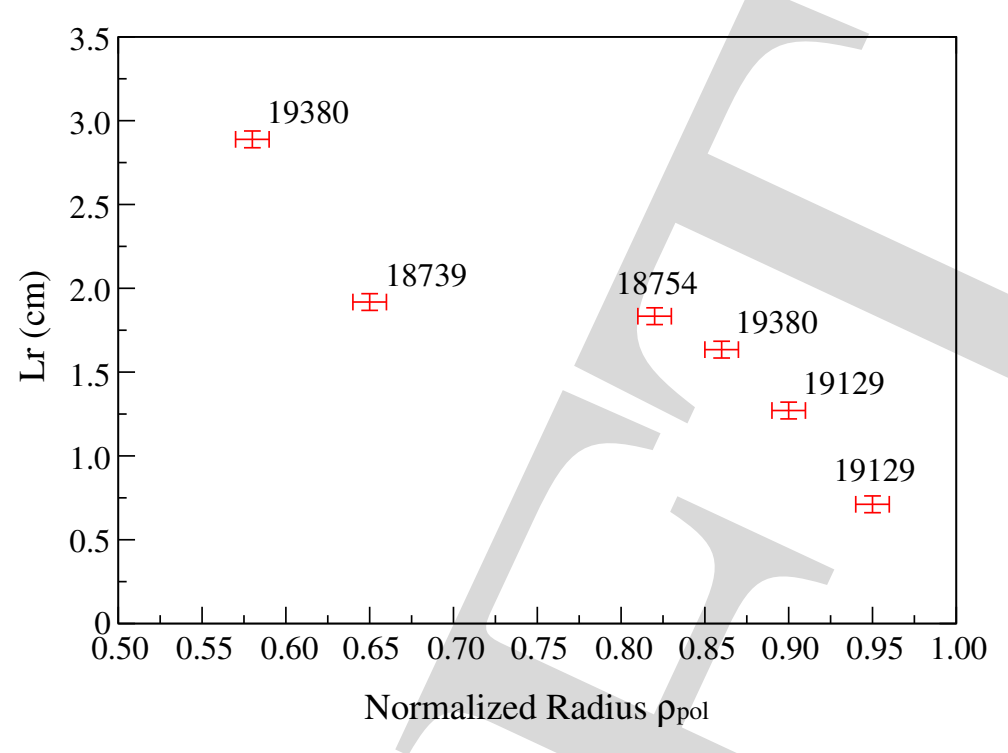

Figure 6. $L_{r}$ measured as a function of normalized radius from a range of L-mode phases in discharges (\#18739-19380). The data was obtained using the correlation Doppler reflectometer system with $O$-mode polarization.

\subsection{Dependence on Plasma Triangularity}

The previous examples of $L_{r}$ measurements suggest a strong link with plasma confinement. Plasma confinement improves with triangularity [23, 24]. To test this relationship, the plasma shape was scanned from low to high triangularity in two similar L-mode upper single null discharges (\#19146 and \#19148) and the results are shown in figure 7. The correlation lengths decrease with increasing triangularity, agreeing with the trend that the confinement improves. This is consistent with previous measurements showing that the radial electric field and its associated shear change with plasma shape [12].

In the experiment, the triangularity ramp also introduces a ramp in the probed wavenumber $k_{\perp}$. The wavenumber is defined by equation 1 where $\theta_{\text {tilt }}$ is the geometric tilt angle between the plasma flux surface normal and the incident microwave beam. Because the antennas on ASDEX Upgrade are fixed, during a triangularity ramp, $\theta_{\text {tilt }}$ changes with a consequent variation in $k_{\perp}$. It is also possible that the change in triangularity affects the turbulence $k$ spectrum. This may also have an influence on the trend shown in figure 7.

The $L_{r}$ dependence on $k_{\perp}$ may be tested in two ways. Firstly, the triangularity dependence may be removed by repeating the experiment with tiltable antennas, allowing the measurement to be performed under constant plasma conditions. This is planned for the following experimental campaign on ASDEX Upgrade when the construction of the new tiltable antennas will be complete [25]. Secondly, the Doppler reflectometer response may be simulated using a 2D Finite Difference Time Domain code with the overall goal to determine the $k_{\perp}$ dependency and test the diagnostic technique for providing $L_{r}$ measurements. The code used along with its results are described in the following section. 


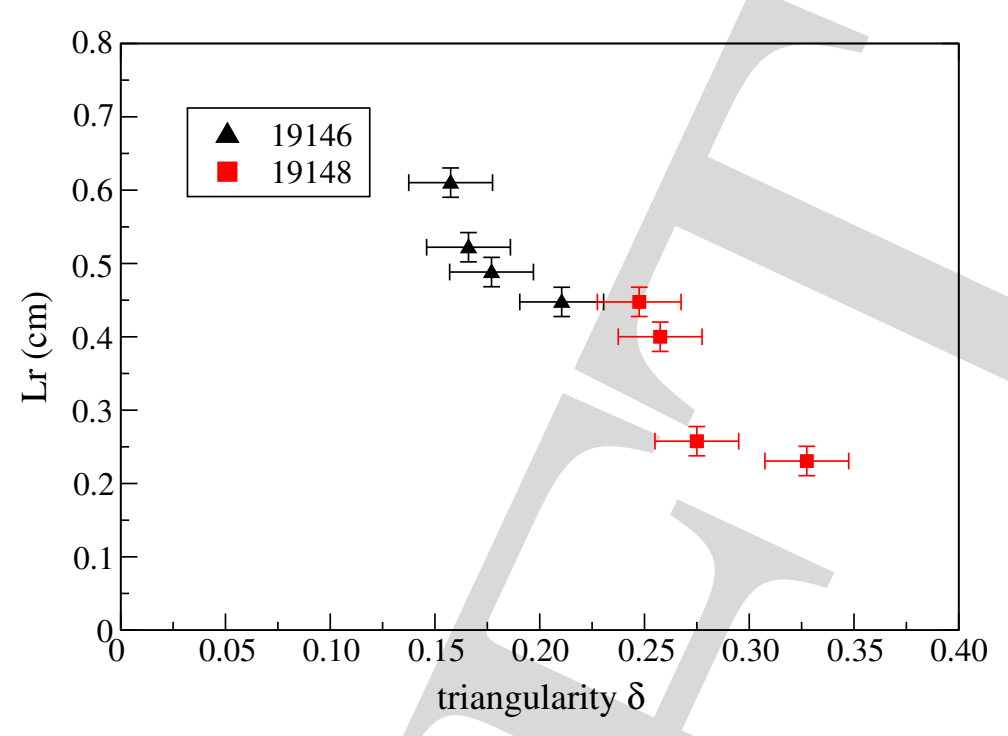

Figure 7. The radial correlation length of the turbulence as a function of plasma triangularity. The measurements were taken in discharges \# 19146 and \# 19148 and show that for increasing triangularity (i.e. increasing confinement), $L_{r}$ decreases.

\section{2D Full Wave Simulation of Doppler Reflectometry}

Modelling of the reflectometer response has been an important aspect of the diagnostic development since the beginning, not only to aid interpretation but also for its predictive capabilities in assisting the design of future devices. Several approaches have been developed, one of the more popular being the two-dimensional full-wave code [26, 27, 28, 29]. As part of this study, a 2D full-wave code in the extraordinary mode has been adapted to simulate Doppler reflectometry in the ASDEX Upgrade tokamak and explore the use of this diagnostic technique for providing $L_{r}$ measurements. To study the dependence of $L_{r}$ on $k_{\perp}$, the simulation offers the freedom to keep all experimental plasma parameters constant while changing only the tilt angle of the launched microwave thereby changing $k_{\perp}$ yet removing any triangularity dependence. The equations in the $2 \mathrm{D}$ full-wave numerical code are presented first, followed by a description of the experimental parameters and modelled turbulence required in the code along with the results of the simulation.

\subsection{The 2D Finite Difference Time Domain Full Wave Code}

For X-mode propagation in a plasma, the Maxwell equations of interest are:

$$
\begin{gathered}
\frac{\partial B_{x}}{\partial t}=-\frac{\partial E_{z}}{\partial y} \\
\frac{\partial B_{y}}{\partial t}=\frac{\partial E_{z}}{\partial x} \\
\mu_{\circ} \varepsilon_{\circ} \frac{\partial E_{z}}{\partial t}=-\mu_{\circ} j_{z}+\frac{\partial B_{y}}{\partial x}-\frac{\partial B_{x}}{\partial y}
\end{gathered}
$$


where the electric field of the wave is in the $\mathrm{z}$ direction and the magnetic field lies in the $\mathrm{xy}$ plane. The constants $\mu_{\circ}$ and $\varepsilon_{\circ}$ are the permeability and permittivity of free space respectively. Note that the current density $\mathbf{j}$ term (defined by $-n_{o}(x) e \mathbf{v}_{\mathbf{e}}$ ) is included to account for the dynamic response of the electrons in the plasma to the electric and magnetic fields introduced by the electromagnetic wave. Under the assumption of a cold plasma $\left(T_{e}=0\right)$, the equation for the current density is:

$$
\frac{\partial j_{z}}{\partial t}=\varepsilon_{\circ} \omega_{p}^{2} E_{z}-\omega_{c} j_{x}+\omega_{c} j_{y}
$$

where $\omega_{p}$ and $\omega_{c}$ are the plasma frequency and cyclotron frequency respectively.

Equations 11 to 14 need to be solved self-consistently. This can be achieved with a finitedifference time-domain (FDTD) algorithm developed by Blaschak and Kriegsmann [30]. An approximate solution to the above Maxwell equations (11-13) is achieved by first discretizing the set of equations into a finite difference rectangular grid of dimensions $(\Delta x, \Delta y)$ and then explicitly integrating the equations forward in time at discrete time steps $(\Delta t)$. In the 2D fullwave code, the variables used are $\Delta x=\Delta y=\lambda_{\circ} / 25$ and $\Delta t=\tau / 25$ where $\lambda_{\circ}$ is the vacuum wavelength of the wave corresponding to a microwave frequency of $61 \mathrm{GHz}$ and $\tau$ is its period. These parameters were chosen as a compromise between computational time and dispersion introduced artificially in the grid from the discretization process [27]. The output of the code includes the Doppler reflectometer In-phase $(I)$ and Quadrature $(Q)$ reflectometer signals.

\subsection{The Input Parameters}

Since the main goal is to determine the dependency of $L_{r}$ on $k_{\perp}$ in discharges \# 19146 and \# 19148, the experimental conditions in those discharges are closely matched in the code. The experimental density, magnetic field, antenna characteristics and a realistic model for the turbulent density fluctuations are used. This section describes the various input parameters of the code and how they are obtained.

4.2.1. Computational Grid As a first step, a computational grid size is carefully optimized. The number of grid points needs to be large enough to include the entire cutoff interaction region and yet be as small as possible to reduce computational time. In the experiment, microwave frequencies were launched between 61.0 and $65.0 \mathrm{GHz}$, corresponding to wavelengths $\lambda$ in the range between $0.49 \mathrm{~cm}$ and $0.46 \mathrm{~cm}$. After a few initial runs of the code, an optimal grid size of 1601 points in the $z$ direction and 1001 points in the $r$ direction was chosen such that the cutoff position for both the low and high triangularity cases of discharges \# 19146 and \# 19148 are included in the grid. The distance per grid point is then $d=\lambda / 25 \approx 0.02 \mathrm{~cm}$. Hence, the computational grid corresponds to roughly $20 \mathrm{~cm}$ in $r$ and 32 $\mathrm{cm}$ in $z$.

4.2.2. Experimental Electron Density and Magnetic Field The electron density $n_{e}$ profile used is a fitted profile to DCN interferometry, core Thomson scattering and standard reflectometry data for discharge \# 19146 at 3.12s. The profile is mapped as density contours 
onto the chosen $r, z$ grid. This density profile is used for all simulations performed. An example of the density profile along $r$ with constant $z=1480$ grid points is shown in figure 8. Likewise, the magnetic field components $B_{\theta}, B_{\phi}$ and $B_{r}$ are obtained from an equilibrium reconstruction code for discharge \# 19146 at $3.12 \mathrm{~s}$ and are also fit to the $r, z$ grid.

4.2.3. Antenna Characteristics The antenna in the $2 \mathrm{D}$ code resembles the X-mode antenna on ASDEX Upgrade. It is modelled as a monostatic system which launches a Gaussian beam distribution with the same beam width as in experiment $(\approx 3 \mathrm{~cm})$. In experiment, the beam is injected upwards at a fixed angle of $45^{\circ}$ to the horizontal. Taking into account the full curvature range of ASDEX Upgrade plasmas, this results in tilt angles $\theta_{\text {tilt }}$ between $16^{\circ}$ and $28^{\circ}$ with respect to the launched microwave beam and the normal to the cutoff surface. In the simulation, with no plasma curvature, to achieve the same range in $\theta_{\text {tilt }}$, the microwave is launched upwards at tilt angles $16^{\circ}$ and $27.5^{\circ}$ to simulate $k_{\perp}$ at the highest and lowest triangularity points in figure 7.

The antenna in the $2 \mathrm{D}$ code is also positioned as close as possible to the plasma to minimize computational time. Outside the plasma, the vacuum equations hold and hence a more simpler and faster code may be utilized for calculating the remaining microwave propagation from the antenna position in the 2D code to the actual antenna position. However, since only the correlation of the fluctuations between two close cutoff layers is of interest, it is permissable to skip the last stage and correlate the signals just outside the plasma boundary. To avoid reflection from the boundaries of the computation grid, a numerical absorber with a depth of 120 grid points is placed on all sides. The antenna is then positioned at the right hand side of the grid in front of an absorber. Hence, the antenna's position is $r=1001-120=881$ grid points, $z=399$ grid points.

4.2.4. Modelling Turbulent Density Fluctuations The reflection layer in the plasma may be modelled as thin 'iso-refractive index' surface with two-dimensional distortions [7]. Each layer has perturbations with a wavenumber k-spectrum in both the radial and poloidal directions. The reflection layer perturbation may be modelled by a Fourier summation given by

$$
\zeta(z, r, t)=\frac{\sigma}{\zeta_{r m s}} \sum^{N} h\left(k_{p}, k_{r}\right) \cos \left(\omega t+k_{p} z+\theta_{p}\right) \cos \left(k_{r} r+\theta_{r}\right)
$$

where $N=231$ is a large prime number to ensure a random distribution, $\sigma$ is the amplitude scale factor, $\zeta_{r m s}$ is the root mean square normalization, $h\left(k_{p}, k_{r}\right)$ is the $k$-spectral profile and $\theta_{p}$ and $\theta_{r}$ are random phases with a uniform distribution between $\pm \pi$. The $k$-spectral profile is modelled here as a top hat function

$$
h\left(k_{p}, k_{r}\right)= \begin{cases}1 & \text { if } 0 \leq k_{p} \geq k_{\text {pmax }}, 0 \leq k_{r} \geq k_{\text {rmax }} \\ 0 & \text { otherwise }\end{cases}
$$

Although equation 15 is a single slice through the $k_{r}$ and $k_{p}$ spectrum, it was found to give random layers with realistic poloidal and radial correlation functions and is computationally 
fast [7]. A simple relationship was found between the 1/e poloidal and radial correlation lengths $\left(L_{p}, L_{r}\right)$ and the spectral widths.

$$
\begin{aligned}
& L_{p}=2.1 / k_{\text {pmax }} \\
& L_{r}=2.1 / k_{r \max }
\end{aligned}
$$

The density fluctuations, $\delta n_{e}$, are calculated by taking a percentage of $\zeta n_{e}$. The percentage is variable, although anywhere between $2-25 \%$ is realistic at the plasma edge. The density distribution modified by the plasma turbulence, via the $\delta n_{e}$ term, is then given by

$$
n_{e}(x, y, t)=\left\langle n_{e}(x, y)\right\rangle_{t}+\delta n_{e}(x, y, t)
$$

where $\left\langle n_{e}(x, y)\right\rangle$ is the average experimental density profile. Figure 8 illustrates for a $7.5 \%$ fluctuation level, the parameters: perturbation $(\zeta)$, density fluctuation $\left(\delta n_{e}\right)$, experimental density $\left(n_{e}\right)$ and the density distribution $\left(n_{e}+\delta n_{e}\right)$ along the $r$ direction with constant $z=1480$ grid points. These parameters are plotted for the case $k_{\text {pmax }} / k_{\circ}=0.76$ and $k_{\text {rmax }} / k_{\circ}=0.15$.

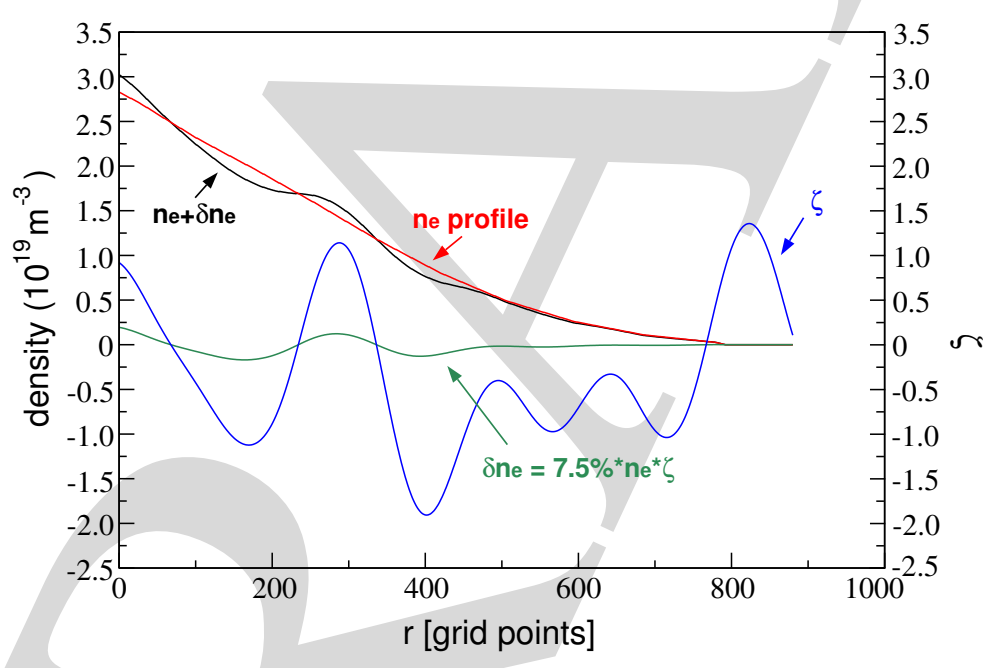

Figure 8. The perturbation, density fluctuation, experimental density profile and density distribution along $r$ with constant $z=1480$ grid points. The density fluctuations are simulated using $k_{\text {pmax }} / k_{\circ}=0.76, k_{\text {rmax }} / k_{\circ}=0.15$ and a $7.5 \%$ fluctuation level.

Using standard correlation techniques [17], the radial and poloidal correlation functions of the model fluctuations may be computed. The results for fluctuations with $k_{\text {pmax }} / k_{\circ}=0.76$ $\left(k_{\text {pmax }}=10 \mathrm{~cm}^{-1}\right)$ and $k_{\text {rmax }} / k_{\circ}=0.15\left(k_{\text {rmax }}=2 \mathrm{~cm}^{-1}\right)$ are shown in figure 9 . Both the poloidal and radial correlation lengths obtained satisfy the relationships given by equations 17 , 18. The poloidal correlation length is $L_{p}=0.248 \mathrm{~cm} \approx(2.1 / 10) \mathrm{cm}$ while the radial correlation length is $L_{r}=1.08 \mathrm{~cm} \approx(2.1 / 2) \mathrm{cm}$. The $k_{r \max }$ is chosen such that the resulting $L_{r}$ is of the same order of magnitude as the $L_{r}$ from experiment.

Another consideration to account for in the modelling of plasma turbulent fluctuations is the speed in which the turbulence moves. (Note the linear dispersion relation $\omega / k_{p}=v_{\theta}$ where $v_{\theta}$ is the propagating poloidal velocity.) If one assumes that the phase velocity of the 

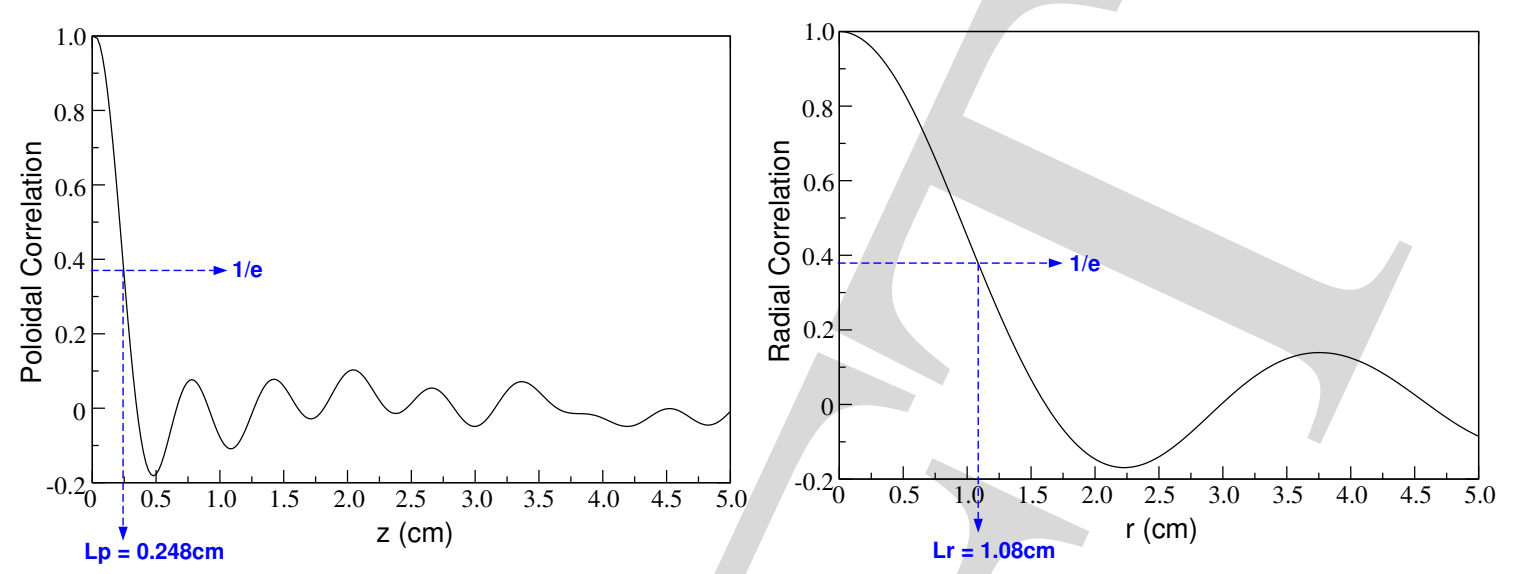

Figure 9. The poloidal and radial correlations of the model fluctuations with $k_{\text {pmax }}=10 \mathrm{~cm}^{-1}$ and $k_{\text {rmax }}=2 \mathrm{~cm}^{-1}$.

turbulence is approximately $4 \mathrm{~km} / \mathrm{s}$ in the poloidal direction (i.e. $z$ direction), it is obvious that the plasma rotation velocity should be increased in order to make simulations in a short period of time. In [31], it was shown that the velocity can be increased up to $c / 20$ without additional effects, where $c$ is the speed of light. In the initial testing phase of the code, it was found that turbulence movements of +30 grid points in $z$ gave acceptable I and Q output signals. Steps of +1 were statistically oversampled (the I and Q signals were perfect sine waves) while steps greater than +100 appeared undersampled. As a consequence, a grid size of 1001 points in $r$ and 12552 points in $z$ of the displacement fluctuations was used. The movement of the turbulence is then modelled as the original computational grid size (i.e. 1001 by 1601 grid points) is stepped up +30 grid points in this larger sized grid.

\subsection{Procedure for Obtaining $L_{r}$}

There is a minor difference in the method used in the experiment and simulation to determine $L_{r}$. In the experiment, a large amount of data is available $(20 \mathrm{MHz}$ sampling rate) and the coherence spectra is employed. From the 1/e coherence, $L_{r}$ is extracted. In the simulation, however, due to computational time, only 128 data points are computed. $L_{r}$ is obtained here from the 1/e radial cross correlation decay. The correlation can be computed with far less data points, although the statistical error goes as $1 / \sqrt{N}$ where $\mathrm{N}$ is the number of data points. Nevertheless, the coherence and correlation contain the same information and the same $L_{r}$ is obtained just with different accuracies.

The experimental points simulated by the full wave code are just the two extreme $k_{\perp} / k_{\circ}$ cases (i.e. $k_{\perp} / k_{\circ}=0.47$ and 0.69 ), which represent the range currently possible on ASDEX Upgrade. In the correlation experiment, one frequency channel was held constant at $61.0 \mathrm{GHz}$ while the other frequency channel was stepped away to a maximum of $65.0 \mathrm{GHz}$. By 63.0 $\mathrm{GHz}$, the complex coherence of the signals $\left(I_{1}+i Q_{1}\right.$ and $\left.I_{2}+i Q_{2}\right)$ generally drops to its $1 / \mathrm{e}$ value. In the simulation, eight frequencies were selected between 61.0 and $65.0 \mathrm{GHz}(61.0$, $61.1,61.3,61.5,61.7,62.1,63.0$ and $65.0 \mathrm{GHz})$. The code is run for each of these microwave 
launch frequencies until $128 \mathrm{I}$ and $128 \mathrm{Q}$ output values are collected. 128 was chosen to save computational time and yet offer an acceptable statistical error of $1 / \sqrt{128}=8.8 \%$. The I and Q signals are then cross-correlated $\left(I_{1}\right.$ with $I_{2}$ and $Q_{1}$ with $\left.Q_{2}\right)$ for varying frequency differences, $\Delta f$. [The eight frequencies selected for the simulation offer twenty possible combinations of $\Delta f$.]

4.3.1. Electric Field Patterns The 2D full wave code provides electric field patterns which are used to ensure the optimum settings of the code and check for a reasonable output from the simulations. An example set of patterns is presented in figure 10. A microwave beam of $65.0 \mathrm{GHz}$ is launched into the plasma with a fixed fluctuation pattern (i.e. not moving) but varying fluctuation level from $0 \%$ to $7.5 \%$. Approximately 2000 iterations or time steps $(\Delta t)$ are needed for the microwave beam to propagate into the plasma, interact at the cutoff layer with the density fluctuations and for the information of the interaction to return to the monostatic antenna. After producing electric field plots and examining the output $I$ and $Q$ signals, it was decided to move the turbulence +30 grid points in $z$ every 4000 iterations before sampling the I and Q signals. 4000 iterations is a good compromise between the time needed for the signals to become relatively stable and yet not too long of a waiting time to obtain a result in a reasonable amount of time. 4000 iterations takes approximately one hour of computation time on a Sun-Fire workstation with processor speed of $1.6 \mathrm{GHz}$ and RAM memory of 16GB.

The first example of an electric field plot with a $0 \%$ fluctuation level, shown in figure 10 , relates to the case of a plasma with no turbulence. As soon as the turbulence is increased via the density fluctuation level, side lobes appear in the electric field patterns. The interaction between the microwave beam and the turbulence at the cutoff layer becomes more complicated at the higher fluctuation levels.

4.3.2. Cross-Correlation The modelled turbulence may be altered by varying three parameters: level of density fluctuations and the normalized spectral widths $k_{\text {pmax }} / k_{\circ}$ and $k_{\text {rmax }} / k_{\circ}$. One of the first simulation cases performed was with a turbulence defined by a $5 \%$ fluctuation level, $k_{\text {pmax }} / k_{\circ}=0.76$ and $k_{\text {rmax }} / k_{\circ}=0.15$. By cross-correlating the resulting I and Q output signals (made up of 128 points each) for the set of 8 frequencies, one obtains plots of the I and Q cross-correlations varying with frequency separation. The frequency separation $\Delta f$ may be translated to a radial separation $\Delta r$ with the use of the $\mathrm{X}$-mode cutoff equations (given in reference [12]) such that the cross-correlations may be plotted as a function of $\Delta r$ as shown in figure 11. As expected for homodyne reflectometer signals, the radial correlation shows a decaying oscillatory structure versus radial separation due to the sine/cosine dependence of the homodyne I and Q signals on the phase. An envelope may be fitted to the maxima to give the correct decay [8]. In this example, a Laplacian is fitted to the correlation, giving $L_{r}=0.9 \mathrm{~cm}$. 


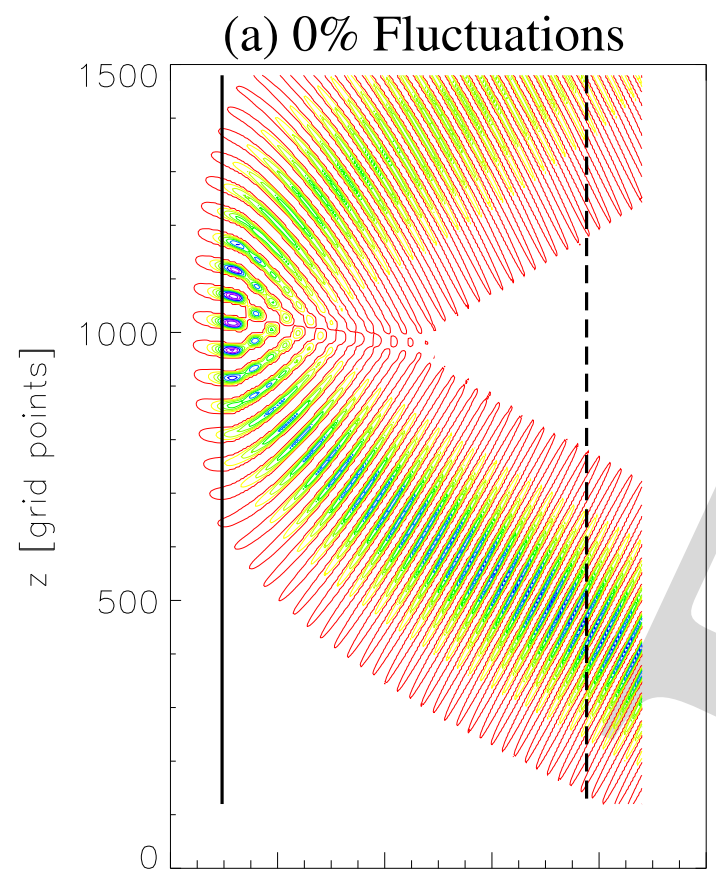

(c) $5 \%$ Fluctuations

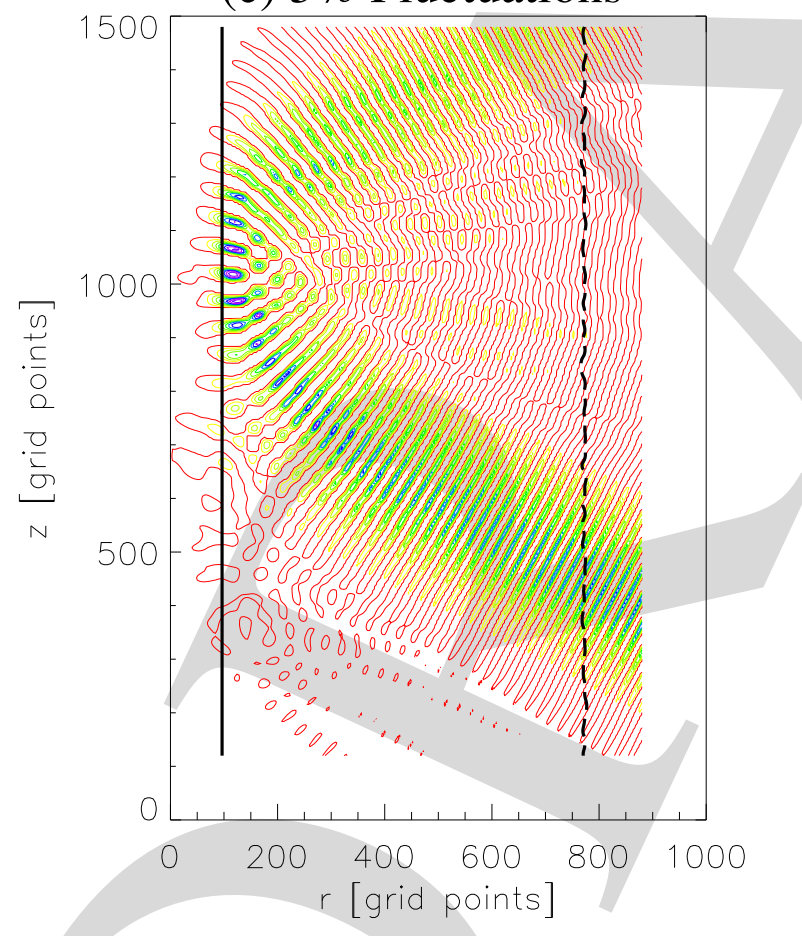

(b) $2.5 \%$ Fluctuations

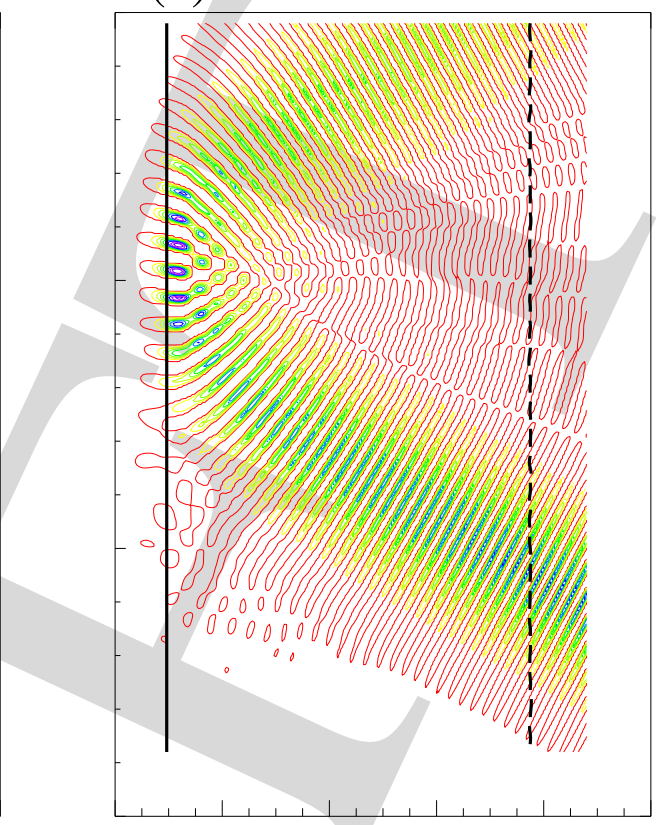

(d) $7.5 \%$ Fluctuations

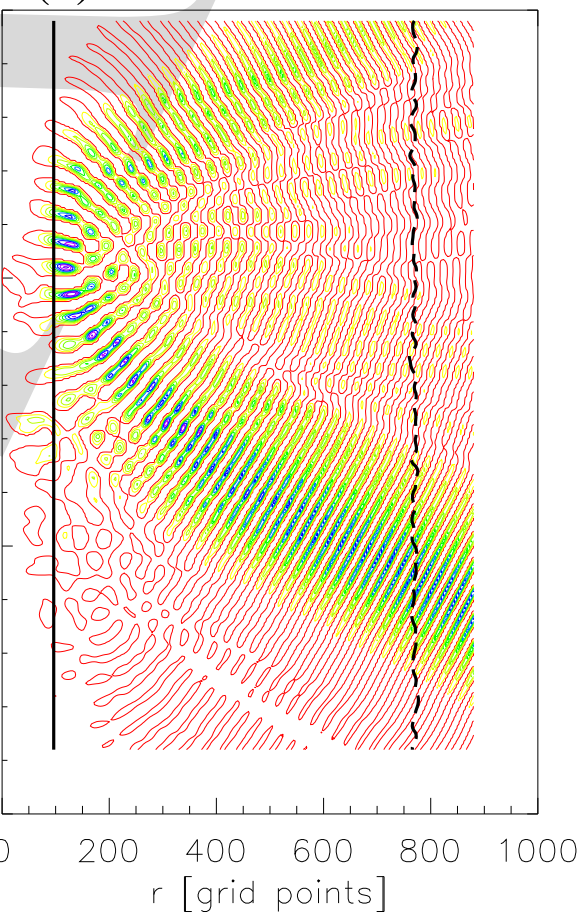

Figure 10. Electric field patterns simulated for $k_{\text {pmax }} / k_{\circ}=0.76, k_{r \max } / k_{\circ}=0.15$ with various density fluctuation levels. The microwave is launched in X-mode at an upwards 27.5 degree angle $\left(k_{\perp} / k_{\circ}=0.69\right)$ with $65.0 \mathrm{GHz}$ frequency. The dashed line indicates the plasma boundary while the solid line represents cutoff.

\subsection{Simulation Results}

The simulation results are presented as a series of scans in the normalized radial wavenumber $k_{\text {rmax }} / k_{\circ}$, the normalized poloidal wavenumber $k_{\text {pmax }} / k_{\circ}$ and the density fluctuation level 


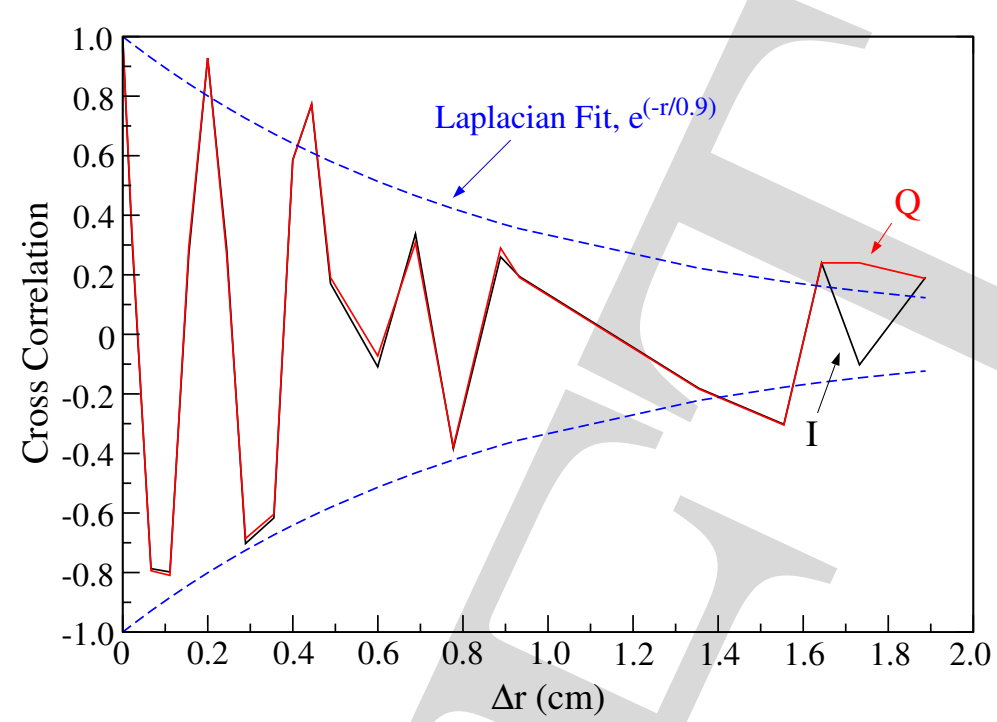

Figure 11. Cross-correlation of the output $I$ and $Q$ signals for $k_{\perp}=9.15 \mathrm{~cm}^{-1}$ case and density fluctuations having $k_{\text {pmax }} / k_{\circ}=0.76, k_{r \max } / k_{\circ}=0.15$ and a $5 \%$ fluctuation level. The Laplacian fit is shown by the dashed curve, from which $L_{r}=0.9 \mathrm{~cm}$.

percentage for $k_{\perp} / k_{\circ}=0,0.47$ and 0.69 . The $k_{\perp} / k_{\circ}=0$ case represents standard reflectometry while the other cases represent the possible range currently measured on ASDEX Upgrade plasmas.

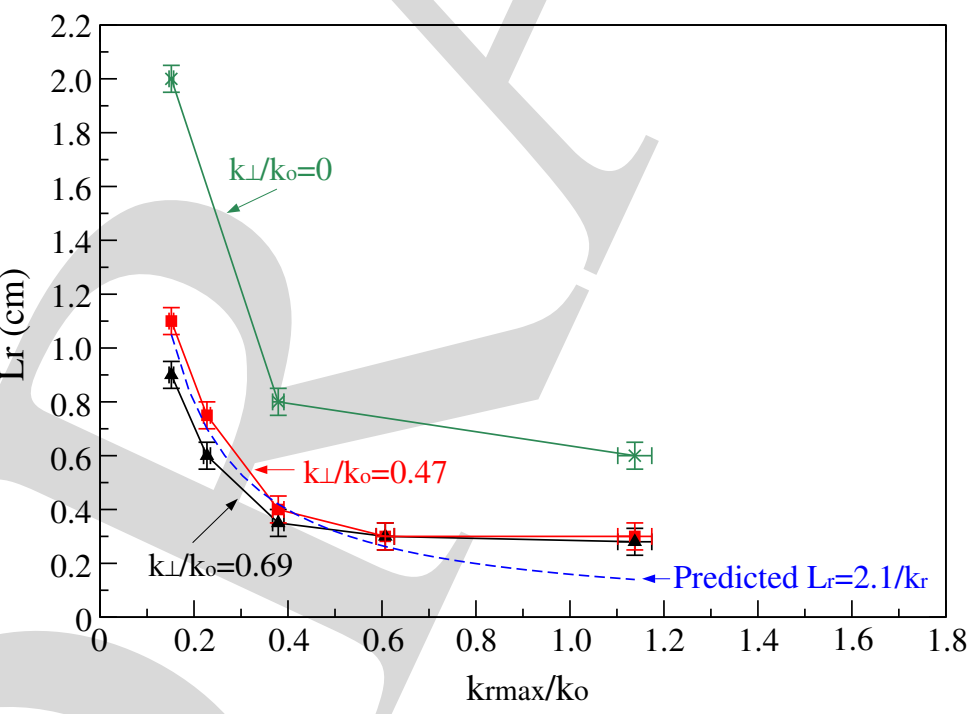

Figure 12. The simulation results during a scan in $k_{r \max } / k_{\circ}\left(k_{p \max } / k_{\circ}=0.76,2.5 \%\right.$ fluctuation level). The dashed curve represents the predicted radial correlation lengths.

Figure 12 shows a scan in $k_{r \max } / k_{\circ}$ with constant $k_{\text {pmax }} / k_{\circ}=0.76$ and a constant $2.5 \%$ fluctuation level. For all non-zero $k_{\perp} / k_{\circ}$ cases, the simulated correlation lengths agree with those predicted by the turbulence model (i.e. $L_{r}=2.1 / k_{\text {rmax }}$ ). This suggests that the diagnostic is capable of measuring the true $L_{r}$. Note that at higher $k_{r \max } / k_{\circ}$ values, the simulated $L_{r}$ starts to diverge from the predicted $L_{r}$, saturating at $L_{r} \approx 0.25 \mathrm{~cm}$. This resolution 
limit of $L_{r}$ measurements will be discussed in the next section. In the $k_{\perp} / k_{\circ}=0$ case, the $L_{r}$ lengths are for all $k_{r \max } / k_{\circ}$ substantially higher than those predicted.

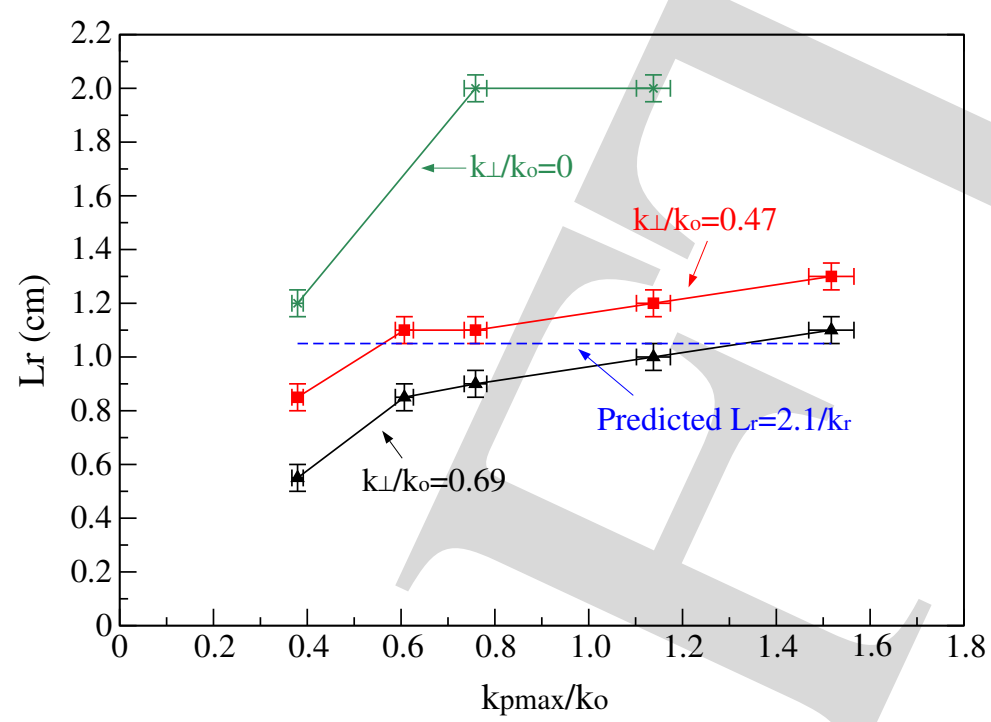

Figure 13. The simulation results during a scan in $k_{p \max } / k_{\circ}\left(k_{\text {rmax }} / k_{\circ}=0.15,2.5 \%\right.$ fluctuation level). The dashed line represents the predicted radial correlation lengths.

Figure 13 shows that $L_{r}$ increases only slightly over a large variation in $k_{\text {pmax }} / k_{\circ}$, suggesting that $L_{r}$ is independent of $k_{p m a x} / k_{\circ}$. In addition, only a small difference between the nonzero $k_{\perp} / k_{\circ}$ cases is seen with both lying close to the predicted $L_{r}$. When $k_{\perp}>k_{\text {pmax }}$ (i.e. trying to probe beyond the model turbulence k-spectrum), then no sensible $L_{r}$ is obtained as shown in the figure by a drop in the simulated correlation lengths. Again, the simulated $L_{r}$ for zero tilt angle is higher than predicted.

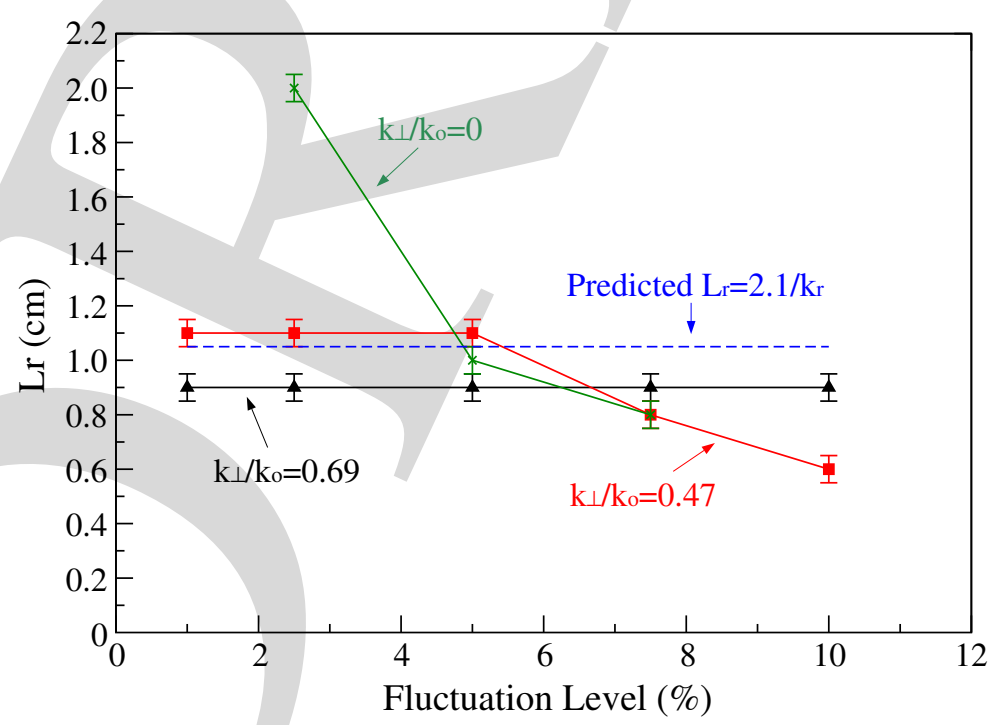

Figure 14. The simulation results during a scan in the turbulence fluctuation level $\left(k_{\text {pmax }} / k_{\circ}=\right.$ $\left.0.76, k_{r \max } / k_{\circ}=0.15\right)$. The dashed line represents the predicted radial correlation lengths.

Simulations were also performed for constant $k_{\text {pmax }} / k_{\circ}=0.76$ and $k_{r \max } / k_{\circ}=0.15$ while 
the fluctuation level was varied from 1 to $10 \%$. The results are illustrated in figure 14 for three $k_{\perp} / k_{\circ}$ cases with the predicted correlation length indicated by the dashed line. For the nonzero wavenumber cases, the $L_{r}$ obtained are similar and close to those predicted at low fluctuation levels. However, by $7.5 \%$ fluctuation level, $L_{r}$ drops in the $k_{\perp} / k_{\circ}=0.47$ case. Similarly, for $k_{\perp} / k_{\circ}=0$, the simulated lengths are first much higher than the predicted lengths and then decrease to lower values. This has been seen previously by $1 \mathrm{D}$ and 2D simulations of standard reflectometry showing that at low fluctuation levels, the simulated $L_{r}$ is larger than predicted while at high fluctuation levels, the simulated $L_{r}$ is smaller [7, 32].

\subsection{Discussion}

The simulation results show that Doppler reflectometry is a specialized technique which may be used for correlation length measurements. The simulated correlation lengths agree well with the correlation lengths of the modelled turbulence (i.e. the predicted ones). Furthermore, $L_{r}$ does not appear to be dependent on $k_{\perp}$; the simulated $L_{r}$ for the two nonzero $k_{\perp}$ cases have roughly the same shape and magnitude in all three scans. The simulated $L_{r}$ is similar to the turbulence model $L_{r}$ in the ranges: $0.15 \leq k_{\text {rmax }} / k_{\circ} \leq 0.60,0.60 \leq k_{\text {pmax }} / k_{\circ} \leq 1.52$ and $1 \% \leq$ density fluctuation level $\leq 7.5 \%$. Hence, it is most likely the change in plasma triangularity which accounts purely for the behaviour shown in figure 7.

In the first scan (i.e. $L_{r}$ versus $k_{r \max } / k_{\circ}$ ), a resolution limit of $L_{r} \approx 0.25 \mathrm{~cm}$ is obtained. This limit has also been observed in experimental data. It is approximately half the vacuum wavelength $\lambda_{\circ}$. Previously, one-dimensional reflectometry models indicated a lower limit of one [33, 34] to four [35] vacuum wavelengths, while two-dimensional models predict slightly improved limits [36, 7, 37]. In the Large Plasma Device (LAPD), radial correlation length measurements by homodyne reflectometry and probe arrays have reached limits below the vacuum wavelength [4]. Hence, it is possible for Doppler reflectometers to measure $L_{r}$ below the vacuum wavelength limit however to determine its exact limit requires further study.

The simulation results indicate a major improvement of Doppler reflectometry over standard reflectometry for providing $L_{r}$ measurements. At normal incidence, when $k_{\perp}=0$, $L_{r}$ is consistently higher than predicted. Standard correlation reflectometry for the use of $L_{r}$ measurements has already been examined using $1 \mathrm{D}$ and 2D simulations. For example, Conway using a 2D physical optics model [7] and Kramer using a 2D full wave code [32] show for $k_{\perp}=0$ that, in general, the simulated reflectometer correlation lengths are not equal to the turbulence correlation lengths, $L_{r}$. For low fluctuation levels, the simulated lengths are larger than the turbulence correlation lengths but fall to below the model $L_{r}$ at high fluctuation levels. Leclert describes a 1D full wave test of radial correlation reflectometry in both linear and nonlinear regimes [38]. In the linear regime (where scattering is weak and the specular component is observable in the reflection spectrum), the reflectometer signal radial correlation lengths can substantially exceed the turbulence correlation length as observed in some experiments [39] and in analytic theory [40]. On the other hand, in the nonlinear regime (at high turbulence levels), correlation reflectometry provides well-defined and highly 
localized turbulence correlation lengths. The nonlinear regime is satisfied by the criterion:

$$
\frac{\omega^{2} L_{r} x_{r}}{c^{2}}\left(\frac{\delta n}{n}\right)^{2} \gg 1
$$

where $\omega$ is the probing microwave frequency, $L_{r}$ is the turbulence radial correlation length, $x_{r}$ is the distance between the plasma and cutoff layer position, $c$ is the speed of light and $\delta n / n$ is the turbulence fluctuation level. Inserting the parameters used in figures 12 and 13, the criterion reduces to $L_{r} \gg 5 \mathrm{~cm}$. Since this is not satisfied in the simulation results, one can assume a linear regime and hence this explains why the standard reflectometry case $\left(k_{\perp}=0\right)$ overestimates $L_{r}$ throughout in both figures. On the other hand, the criterion for nonlinear regimes given in equation 20 reduces to $\delta n / n \gg 6 \%$ for the parameters in figure 14. In figure 14 , we see that at $6 \%$ fluctuation levels, all cases of $k_{\perp}$ provide simulated correlation lengths which agree within error with the model turbulence correlation length. As the fluctuation level increases however and the regime becomes strongly nonlinear, $L_{r}$ becomes underestimated in the simulation for the $k_{\perp} / k_{\circ}=0$ case. It has been observed for standard reflectometry that for fluctuation levels greater than $5 \%$, a divergence between $L_{r}$ determined numerically and analytically begins to appear and increases with fluctuation level [38]. It should be noted that for the highest $k_{\perp} / k_{\circ}$ case simulated here, the reflectometer correlation length continues to agree with the turbulent $L_{r}$ up to a $10 \%$ fluctuation level, suggesting that for high tilt angles, $L_{r}$ measurements from Doppler reflectometry are independent of the turbulent fluctuation level. The implication is that by suppressing the $m=0$ specular component by using a Doppler reflectometer, a consistently more robust measurement of $L_{r}$ is provided compared to standard normal incidence correlation reflectometry. Analytic modelling also indicate the high localization in both linear and nonlinear regimes of the Doppler diagnostic technique $[41,42]$.

\section{Summary}

Correlation Doppler reflectometry was employed for determining the radial correlation lengths of the turbulence on the ASDEX Upgrade tokamak. The experimental measurements agree with theory as well as with experimental measurements from different diagnostics on other tokamak machines. $L_{r}$ decreases by a factor of two at the $\mathrm{L}-\mathrm{H}$ transition at the plasma edge. The negative $E_{r}$ shear was also observed to increase in this plasma region which is consistent with the Biglari, Diamond and Terry (BDT) model [20], which predicts that an increase in absolute shear supresses density fluctuations and stabilizes the turbulence, thereby decreasing $L_{r}$. At the plasma edge, $E_{r}$ shear is high compared with the shear in the core. The $L_{r}$ measurements agree with this observation by increasing monotonically into the plasma core. The correlation lengths were observed to decrease during a plasma triangularity upwards ramp. This experiment motivated a 2D full wave simulation study to determine the relationship of $L_{r}$ with $k_{\perp}$. The simulation employed the experimental density and magnetic fields with a realistic model for the turbulence where the poloidal and radial wavenumbers and fluctuation levels may be altered. The simulation results show that Doppler 
reflectometry measures a consistent and robust radial correlation length. This appears to be due to the introduction of a tilt angle which removes the specular component in the reflected spectrum allowing robust $L_{r}$ measurments in both the linear and nonlinear turbulence regimes. Furthermore, the simulations suggest that $L_{r}$ is independent of $k_{\perp}$. In order to test this dependency experimentally, a repeat of the experiment with constant plasma conditions and a remote steerable antennas system is required, as performed in the simulations.

\section{Acknowledgments}

Dr. Frank Jenko, Dr. Bruce Scott and Dr. Arthur Peeters are acknowledged for discussions regarding $E_{r}$ shear, plasma turbulence and in particular, correlation lengths of the turbulence. Dr. Emanuele Poli and Ms. Carolin Troester are thanked for their support with TORBEAM calculations of the radial cutoff positions and Dr. Christoph Fuchs is gratefully acknowledged for support in fitting density data.

\section{References}

[1] Cripwell P. et al 1991 Proc. $18^{\text {th }}$ European Conf. on Controlled Fusion and Plasma Physics vol 14B Part I. $p 17$

[2] Rhodes T.L. et al 2002 Phys. Plasmas 92141

[3] Kurzan B. et al 2000 Plasma Phys. Control. Fusion 42237

[4] Gilmore M. et al 2000 Plasma Phys. Control. Fusion 42 L1

[5] Hanson G.R. et al 1990 Rev. Sci. Instrum. 613049

[6] Rhodes T.L. et al 1992 Rev. Sci. Instrum. 634661

[7] Conway G.D. 1997 Plasma Phys. Control. Fusion 39407

[8] Estrada T. et al 2001 Phys. Plasmas 82657

[9] Mazzucato E. et al 1993 Phys. Rev. Lett. 711840

[10] Hirsch M. et al 2001 Plasma Phys. Control. Fusion 431641

[11] Conway G.D. et al 2004 Plasma Phys. Control. Fusion 46951

[12] Schirmer J. et al 2006 Nucl. Fusion 46 S780

[13] Schirmer J. et al 2006 33rd EPS Conf. on Controlled Fusion and Plasma Physics (Rome, Italy) P2.136

[14] White R.B. et al 1974 Plasma Physics 16565

[15] Conway G.D. et al $200621^{\text {st }}$ IAEA Fusion Energy Conference (Chengdu, China) IAEA-CN-149/EX/2-1

[16] Schirmer J. et al $200431^{\text {st }}$ EPS Conf. on Controlled Fusion and Plasma Physics (London, England) P4.127

[17] Bendat J.S. et al 1986 Random Data Analysis and Measurement Procedures Wiley-Interscience New York

[18] Rhodes T.L. et al 1998 Plasma Phys. Control. Fusion 40493

[19] Baldzuhn J. et al 1998 Plasma Phys. Control. Fusion 40967

[20] Biglari H. et al 1990 Phys. Fluids $\mathbf{B 2} 1$

[21] Burrell K.H. et al 1992 Plasma Phys. Control. Fusion 341859

[22] Poli E. et al 2001 Comput. Phys. Commun. 13690

[23] Saibene G. et al 2002 Plasma Phys. Control. Fusion 441769

[24] Taylor T.S. et al 1994 Plasma Phys. Control. Fusion 36 B229

[25] Troester C. et al 2006 DPG Frhjahrstagung der Fachverbnde Kurzzeitphysik und Plasmaphysik (VI) 41 P 11.5

[26] Hacquin S. et al 2001 Journal of Computational Physics 1741

[27] Blanco E. et al 2004 Review of Scientific Instruments $\mathbf{7 5} 3822$

[28] Blanco E. et al 2006 Plasma Phys. Control. Fusion 48699

[29] Holzhauer E. et al 2006 Nucl. Fusion 46 S824 
[30] Blaschak J.G. et al 1988 J. Comput. Phys. 77109

[31] Hacquin S. et al 2002 Proc. $29^{\text {th }}$ EPS Conf. on Controlled Fusion and Plasma Physics (Montreux) 26B P5.094

[32] Kramer G.J. et al 2003 Rev. Sci. Instrum. 741421

[33] Hutchinson I.H. 1992 Plasma Phys. Control. Fusion 71225

[34] Gilmore M. 1999 PhD Thesis University of California, Los Angeles

[35] Bretz N. 1997 Phys. Fluids B4 2414

[36] Mazzucato E. et al 1995 Rev. Sci. Instrum. 661237

[37] Bruskin L.G. et al 1997 Fusion Eng. Design 34-35 441

[38] Leclert G. et al 2006 Plasma Phys. Control. Fusion 481389

[39] Nazikian R. et al 2005 Phys. Rev. Lett. 94135002

[40] Gusakov E.Z. et al 2002 Plasma Phys. Control. Fusion 442525

[41] Gusakov E.Z. et al 2004 Plasma Phys. Control. Fusion 461143

[42] Gusakov E.Z. et al 2005 Plasma Phys. Control. Fusion 47959 